\title{
Sistema para Monitoração de Batimentos Fetais para Aplicação em Hospitais Públicos
}

\author{
Ana Vitória M. Inocêncio ${ }^{1}$ orcid.org/0000-0002-1411-7547 \\ Marco A. B. Rodrigues ${ }^{2}$ Dorcid.org/0000-0002-5143-3696 \\ Diogo R. R. Freitas ${ }^{1}$ Borcid.org/0000-0002-5023-699X
}

${ }^{1}$ Escola Politécnica de Pernambuco, Universidade de Pernambuco, Recife, Brasil,
2 Universidade Federal de Pernambuco, Pernambuco, Brasil,
E-mail do autor principal: Ana Vitória Inocêncio anavitoriam @gmail.com

RESUMO

A medicina atual é caracterizada pela busca de métodos de exploração menos invasivos, mais eficientes e que não incomodem tanto o paciente em caso de acompanhamento por períodos longos de tempo. Para superar o risco desnecessário no período de gravidez é importante monitorar a condição cardíaca fetal regularmente, também para identificar possíveis anormalidades. Este trabalho propõe o desenvolvimento de um protótipo para aquisição de sinais de eletrocardiograma fetal ( $\mathrm{EECG}$ ) seguro e que se comunique com outros dispositivos. $\mathrm{O}$ protótipo tem como finalidade a monitorização dos sinais de fECG em pacientes grávidas a partir da vigésima semana de gravidez. Foi desenvolvido um sistema com três canais analógicos de aquisição com filtragem e amplificação. Dois canais foram posicionados sob o abdômen da grávida, alinhados com o umbigo, e o último canal foi adquirido direto do tórax. A conversão analógico-digital foi feita através da placa USB6009 e o processamento do sinal foi feito através do software Matlab. O sistema foi testado em quatro mulheres grávidas voluntárias do Hospital das Clínicas da UFPE, e o algoritmo desenvolvido foi verificado com o auxílio de um médico especialista que marcou os pontos com o auxílio do data cursor do Matlab. A diferença entre a frequência média dos batimentos fetais medida pelo sistema proposto e a contagem manual realizada pelo médico especialista foi 0,94 bpm, que representa um erro de 0,7\%.

PALAVRAS-CHAVE: Dispositivo Portátil, ECG fetal, Processamento de sinais

\section{ABSTRACT}

Modern Medicine is characterized by the search for less invasive methods, more efficient and which offers comfort for long time attendance. To overcome unnecessary risk during the period of pregnancy, it is important to monitor fetal heart condition regularly, also to identify possible abnormalities. This paper proposes a safe acquisition device for fetal electrocardiography (fECG) with a communication channel. The device purpose is monitoring fECG in pregnant patients after the twentieth week. A system was developed with three analog channels for data signal acquisition, filters, and amplification. Two of the canals were positioned over the abdomen of the pregnant woman, aligned with the belly button, and the last channel was acquired directly from the thorax. The analog-digital conversion was made using the USB6009 board and the signal processing with the Matlab software. The system was tested in four volunteer pregnant women from Hospital das Clínicas of the UFPE and the algorithm was verified by a specialist physician through the Matlab data cursor.The difference between the mean frequency of fetal heart rate measured by the proposed system and the manual counting performed by the specialist physician was 0,94 bpm, which represents an error of 0,7\%.

KEY-WORDS: Portable Device, fetal ECG, Signal Processing 


\section{INTRODUÇÃO}

A captura de sinais bioelétricos pode ser realizada de forma invasiva e não invasiva. Embora os métodos invasivos normalmente permitam a aquisição do sinal com menos interferências, é sempre preferível utilizar-se de métodos de aquisição não invasivos que proporcionem as mesmas conclusões/diagnósticos dos métodos invasivos $[\mathbf{1}, \mathbf{2}]$.

O período de gravidez é de vital importância no diagnóstico e prevenção de problemas do feto que podem surgir por adversidades. As mulheres nesta condição devem ser monitoradas regularmente a partir do segundo trimestre da gravidez, estágio em que os batimentos fetais começam a ser mais facilmente percebidos [3].

As mudanças na frequência cardíaca fetal estão associadas com o risco de complicações na gravidez que o feto está correndo, sendo um dos fatores que determina o momento em que o médico deve intervir. A importância da determinação dos batimentos fetais durante o trabalho de parto é justificada em função da possibilidade feto entrar em sofrimento por um déficit de oxigênio. Neste caso, é recomendado a ausculta dos batimentos fetais a cada meia hora para identificar e assim diminuir as chances de o feto ter complicações [4].

Em geral, hospitais públicos, dentre eles os hospitais universitários, carecem de profissionais de saúde para monitorar a demanda de algumas dezenas de mulheres grávidas em trabalho de parto [5]. Um sistema automatizado que possibilite a medição dos batimentos fetais oferece uma opção para auxiliar os profissionais de saúde nesse monitoramento.

Um método utilizado para avaliar a situação do feto é a cardiotocografia (CTG), que consiste em um exame que monitora a frequência dos batimentos fetais $e$ as contrações uterinas durante um dado intervalo de tempo, em geral de 10 a 20 minutos. Existem quatro métodos para realizar medições CTG: a magnetocardiografia (MCG) e a fonocardiografia (PCG), utilizando o efeito Doppler através do ultrassom e o eletrocardiograma (ECG).

A magnetocardiografia tem sua limitação no tamanho e custo do equipamento [6]. A amplitude do sinal adquirido através da fonocardiografia é cerca de dez vezes menor do que a amplitude materna e não tem uma boa relação sinal-ruído $[3,7]$. A técnica que utiliza o 2 efeito Doppler é mais confiável e largamente aceita para interpretar as mudanças na frequência das ondas sonoras refletidas pela pulsação nos vasos fetais, contudo, dependendo da posição do feto, aparecem muitas limitações na aquisição dos dados durante várias horas [8]. Além disto, não é indicado o uso por um tempo prolongado.

O sinal de Eletrocardiograma (ECG) é resultante do sinal elétrico da atividade muscular do miocárdio. A Figura 1 ilustra o traçado clássico do sinal de ECG, composto pela onda $\mathrm{P}$, O complexo QRS e pela onda T. O fECG (Eletrocardiograma fetal) é captado por meio de eletrodos, situados normalmente no abdômen da paciente [9, 10]. O ECG possibilita 0 monitoramento do QRS fetal (fQRS). Infelizmente, os sinais ECG fetal adquiridos de forma não invasiva geralmente são corrompidos por muitas fontes de ruído interferentes, mais significativamente pelo eletrocardiograma materno (MECG), cuja amplitude é geralmente maior do que a do ECG fetal [11].

Monitorar o fECG permite obter informações importantes sobre a condição do feto durante a gravidez. Através de eletrodos dispostos sobre a barriga da mãe, é possível medir de maneira não invasiva, os sinais elétricos gerados pelo coração do feto. Contudo, neste método de aquisição, o sinal cardíaco fetal tem uma amplitude cerca de dez vezes menor do que o sinal materno e com mais artefatos, como a componente DC resultante da interface pele-eletrodo, o ruído dos eletrodos, entre outros[3].

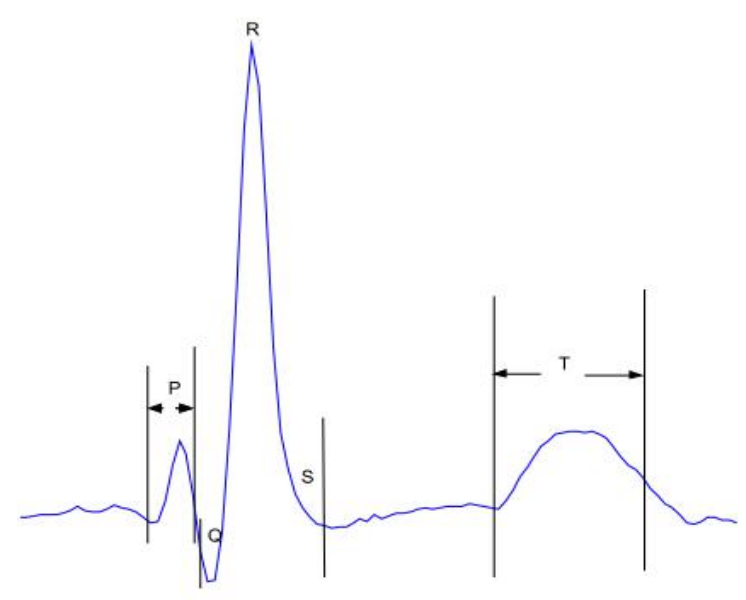

Figura 1: Traçado clássico do ECG. Fonte: [12]. 
Diante dos fatos apresentados, esta pesquisa teve como objetivo o desenvolvimento de um sistema que realize a aquisição do sinal abdominal e a monitoração dos batimentos fetais.

\section{MATERIAIS E MÉTODOS}

O desenvolvimento do protótipo teve duas etapas. A primeira etapa consistiu no desenvolvimento de um circuito analógico para aquisição e tratamento analógico do sinal. A segunda etapa consistiu no processamento digital do sinal.

O protótipo desenvolvido possui três canais de ECG. Os eletrodos foram dispostos conforme a Figura 2, sendo posicionados sobre o abdômen e tórax da gestante através de eletrodos descartáveis, visando a aquisição dos sinais correspondentes aos dois hemisférios e ao tórax materno. Os eletrodos de GND foram fixados nas costas da voluntária.

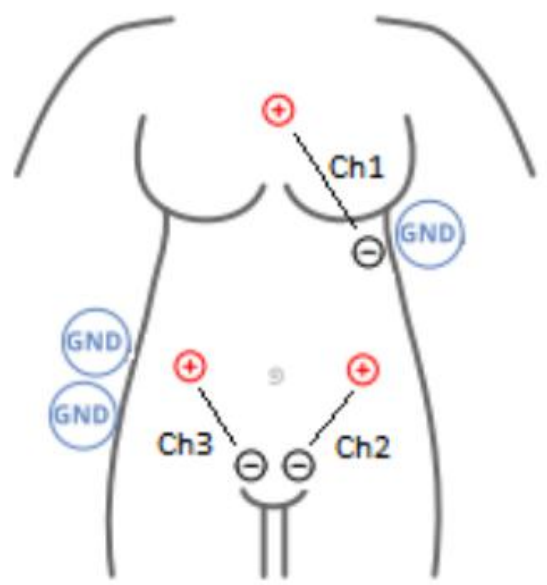

Figura 2:Disposição dos eletrodos. Fonte: Acervo do autor.

A coleta foi realizada em quatro voluntárias do Hospital das Clínicas da UFPE, segundo o posicionamento dos eletrodos conforme a Figura 2. Foram realizadas 3 coletas por voluntária durante um período maior do que 10 segundos.

Para o estágio inicial de amplificação, foi utilizado o amplificador de instrumentação INA129. O amplificador de instrumentação é um arranjo de amplificadores operacionais e tem como principal finalidade à amplificação do sinal diferencial. O INA129 é muito aplicado na aquisição de biossinais a partir de eletrodos externos não- invasivos. Os amplificadores de instrumentação têm como benefício sua alta rejeição a sinais de modo comum em comparação com amplificadores de uso geral, se tornando ideais para sinais com baixa relação sinal-ruído, como o ECG abdominal. A Figura 3 mostra a topologia clássica do amplificador de instrumentação. A partir da mudança do valor do resistor $R_{g}$ é possível modificar o ganho do sinal de saída. Neste trabalho, foi utilizado $\mathrm{Rg}=100 \Omega$ (ganho aproximado de 500X) [13].

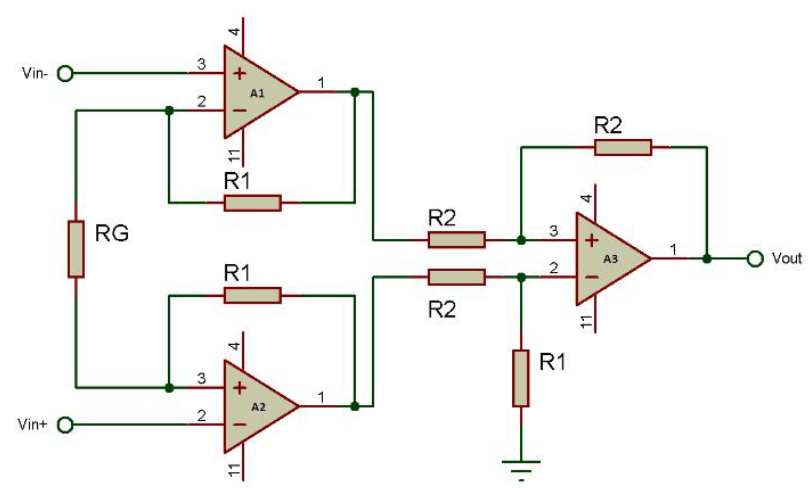

Figura 3: Topologia clássica do amplificador de instrumentação.

Fonte: Acervo do autor.

Após a amplificação inicial do sinal, é necessário retirar algumas frequências indesejadas do sinal para otimizar a conversão analógico-digital (AD). Um filtro é um circuito capaz de permitir a passagem de determinadas frequências e bloquear as demais, atuando como um tipo de seletor de frequência [14].

Para o trabalho apresentado, foi construído um filtro do tipo passa-banda resultante da concatenação de dois filtros, um passa-alta e outro passa-baixa. O filtro que permite apenas a passagem das altas frequências é passivo de primeira ordem e sua frequência de corte foi projetada para $1 \mathrm{~Hz}$, visando a eliminação do offset do sinal e centralização no eixo horizontal.

O principal objetivo deste circuito é eliminar o efeito causado na interface pele-eletrodo, e atenuar as componentes resultantes da movimentação do paciente, inclusive da respiração. Optou-se por projetar um filtro passabaixa passivo seguido de um amplificador com realimentação negativa para compensar a atenuação do sinal devido aos dois filtros passivos em cascata. A frequência de corte do filtro passa- 
baixa (PB) foi projetada para aproximadamente $450 \mathrm{~Hz}$ por conta do espectro de frequências que compõe o sinal de ECG [3]. O valor da frequência de corte foi escolhido pelo fato da frequência de amostragem ser de $1000 \mathrm{~Hz}$ e pelo teorema da amostragem de Nyquist-Shannon[15]. Após a filtragem, o sinal foi amplificado pelo fato dele ter sido atenuado pelos filtros passivos. A Figura 4 ilustra os filtros projetados e o amplificador nãoinversor.

Após a filtragem do sinal, foi projetado um somador não inversor utilizando um amplificador operacional. Este circuito teve como objetivo adicionar um offset de $2,5 \mathrm{~V}$ no sinal para condensá-lo entre $+1 \mathrm{~V}$ e $4 \mathrm{~V}$.

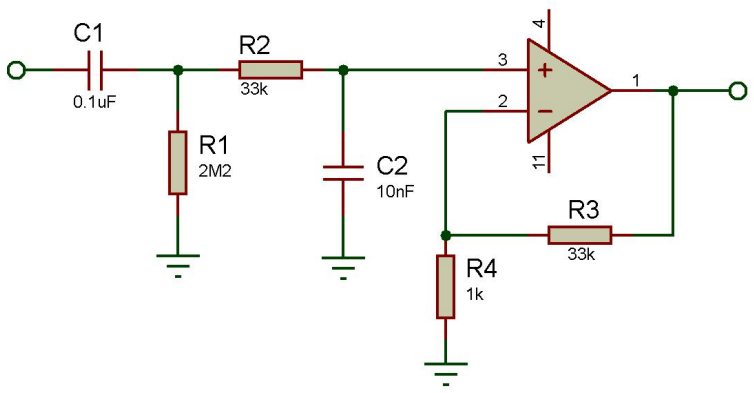

Figura 4: Filtros projetados.

Fonte: Acervo do autor.

A conversão $A D$ foi realizada com o auxílio da placa USB6009 da NationalInstruments. Este sistema oferece funções básicas de aquisição de dados para uma aplicação simples, além de ser considerado um produto de baixo custo pelo fabricante [16].A placa se comunica com o computador por USB, através do software Signal Express (versão 15.0.0). A resolução da entrada analógica é de 14 bits no modo diferencial, contudo o sinal de entrada podendo estar no intervalo de $-10 \mathrm{~V}$ a $10 \mathrm{~V}$, com acurácia de 7.73 $\mathrm{mV}$ [17]. O diagrama de blocos da Figura 5 mostra o fluxo do sistema.

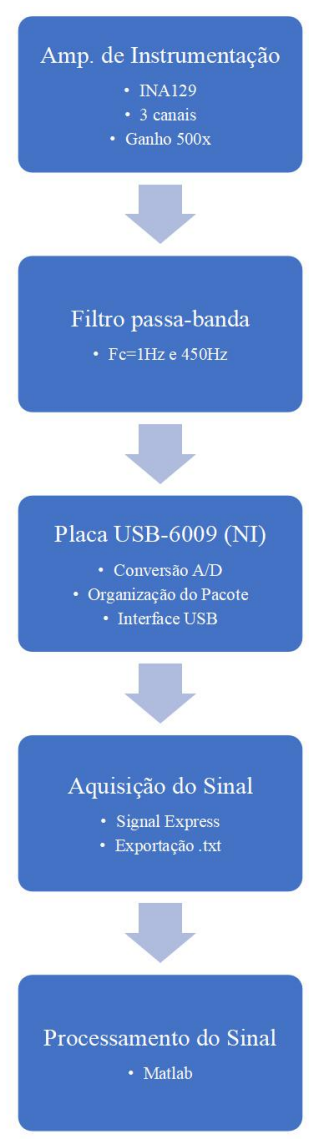

Figura 5:Diagrama de blocos do sistema Fonte: Acervo do autor.

Os dados gravados no software Signal Express da National Instruments foram exportados para serem processados no software Matlab ${ }^{\circledR}$ (versão R2015a). Assim, foi realizada a leitura dos dados contendo os pontos referentes ao canal trabalhado e ao tempo decorrido. As operações de processamento digital dependem diretamente de algumas propriedades dos sinais, onde foram definidos alguns parâmetros, tais como a frequência de amostragem e o comprimento do sinal. Em seguida, foram aplicadas técnicas de processamento com a finalidade de eliminar o batimento cardíaco materno e calcular a frequência cardíaca fetal. O fluxograma completo do processamento de sinais pode ser visto na Figura 6. É válido salientar que este protótipo visou a aquisição do sinal e a elaboração do seu processamento para futuramente embarcar em apenas um dispositivo portátil. 


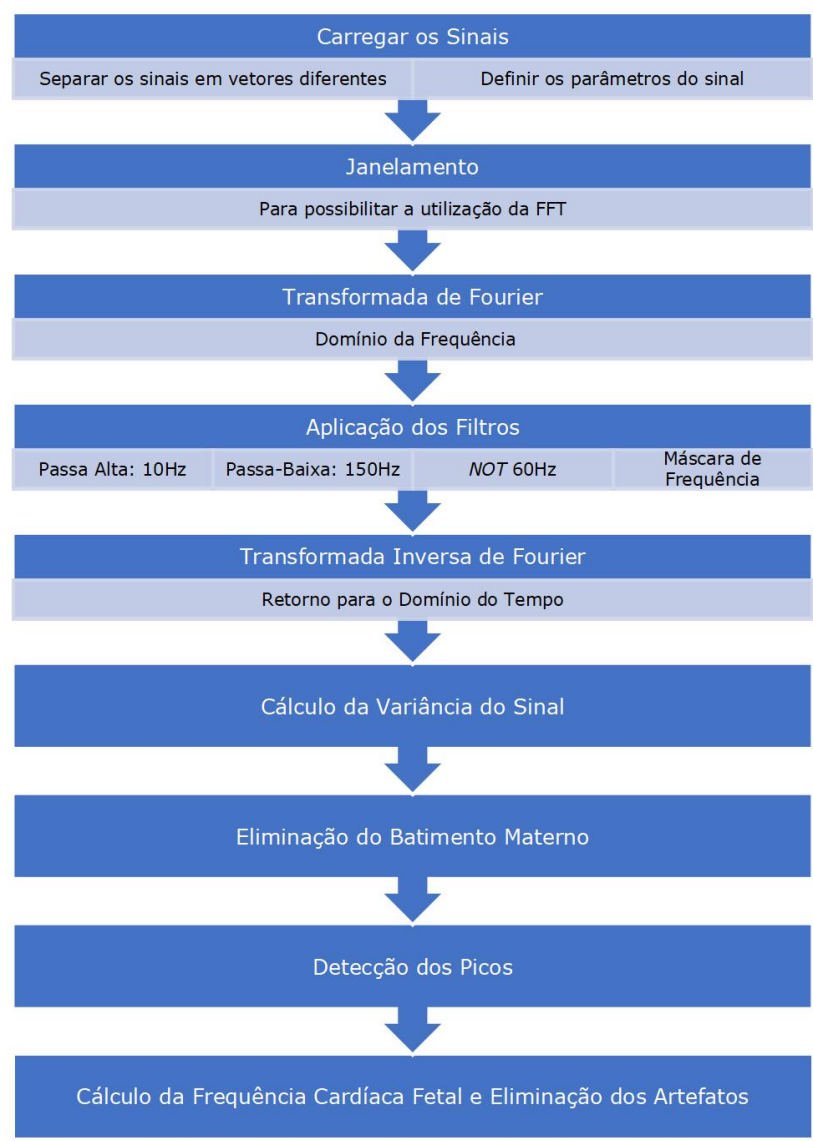

Figura 6:Etapas do processamento dos sinais Fonte: Acervo do autor.

A aplicação da transformada de Fourier de tempo curto está intrinsecamente ligada ao teorema da amostragem. Para a implementação da transformada de Fourier, o sinal precisou ser dividido em janelas com o número de pontos igual à frequência de amostragem (Fs), para que a função no software $M_{a t l a b}{ }^{\circledR}$ possa calcular a transformada de zero à $\mathrm{Fs} / 2$ [15].

Logo após o janelamento, foi aplicada a transformada rápida de Fourier (FFT, Fast Fourier Transform) nas janelas. Foi criada uma máscara de frequências para retirar apenas as componentes de frequência indesejadas do sinal. Desta maneira, a matriz da máscara de frequências criada eliminou as seguintes componentes:

- $\mathrm{OHz}-10 \mathrm{~Hz}$ (Filtro Passa-Alta): esta faixa de frequência corresponde à componente $D C$ do sinal, às pequenas flutuações resultantes da respiração e à onda T do ECG Materno;

- $59 \mathrm{~Hz}-61 \mathrm{~Hz}$ (Filtro NOT): correspondente ao ruído de $60 \mathrm{~Hz}$ resultante da rede elétrica brasileira;
- $150 \mathrm{~Hz}-500 \mathrm{~Hz}$ (Filtro Passa-Baixa): para retirar as altas frequências e os harmônicos de $180 \mathrm{~Hz}, 240 \mathrm{~Hz}$ e $300 \mathrm{~Hz}$. Estes harmônicos são resultado da frequência da rede elétrica e da iluminação $(60 \mathrm{~Hz})$ [18].

Após a realização desta operação, as frequências indesejadas foram eliminadas e a transformada inversa de Fourier foi aplicada ao sinal.

Para a identificação do batimento materno, foi utilizada a ferramenta estatística chamada variância. Como o batimento materno apresenta uma amplitude maior do que o batimento fetal, a variância calculada entre os dois sinais, necessariamente, apresentará um pico durante o complexo QRS materno (Figura 1). Após encontrados os picos referentes aos batimentos cardíacos maternos, foi definida uma linha de base na região, visando sua eliminação.

Para o algoritmo de detecção de picos, primeiramente, são separadas as regiões do gráfico que contém amplitudes correspondentes a $60 \%$ do valor máximo do vetor. Após isto, o algoritmo entra em um comando de repetição que detectará os picos locais dentre os valores que foram separados. Feito isto, o programa guarda em uma matriz os valores dos picos locais e sua posição no tempo.

Após a detecção dos picos correspondentes aos batimentos fetais, é feito o cálculo da frequência cardíaca fetal média ( $f c f m$ ) e do desvio padrão $(\sigma)$. Desta forma, foi realizado a eliminação dos sinais de ECG com os batimentos abaixo $(<\mathrm{fcfm}-\sigma)$ ou acima $(>\mathrm{fcfm}+\sigma)$ dos limites definidos.

\section{RESULTADOS E DISCUSSÃo}

A coleta de dados foi realizada segundo o projeto aprovado no comitê de Ética da Universidade Federal de Pernambuco, projeto da plataforma Brasil com número CAAE 61990316.4.0000.5208. Foram feitas coletas em 4 pacientes. A Figura 7 ilustra o período de 3 segundos de uma das amostras coletadas. 


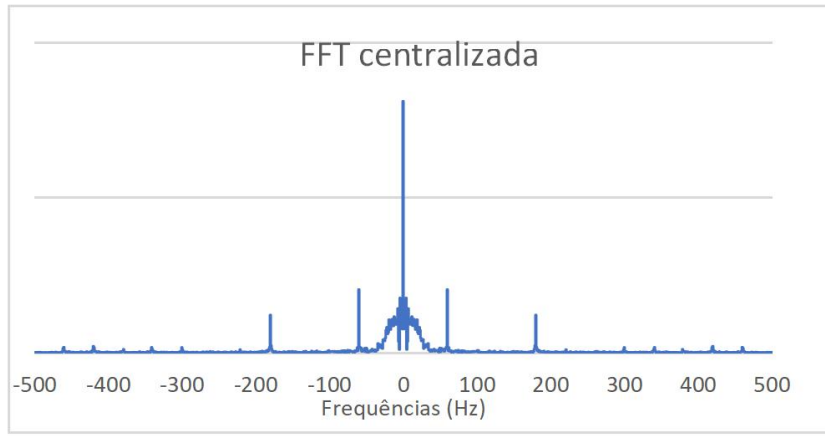

Figura 7: Sinal adquirido sem processamento. Os círculos vermelhos indicam o batimento fetal.

Fonte: Acervo do autor.

A análise do espectro de frequência do sinal adquirido pode ser vista na Figura 8. Nesta análise pode-se observar que o ruído de $60 \mathrm{~Hz}$ e seus harmônicos estão presentes no sinal e com uma amplitude consideravelmente alta, principalmente o terceiro harmônico, situado em $180 \mathrm{~Hz}$. A máscara utilizada no processo de filtragem foi capaz de retirar do sinal a tensão de offset e as pequenas ondulações, além das frequências com mais de $150 \mathrm{~Hz}$ e também os harmônicos de $60 \mathrm{~Hz}$. A Figura 9 ilustra a máscara de filtragem utilizada e a Figura 10 mostra o sinal antes e após a aplicação da mesma.
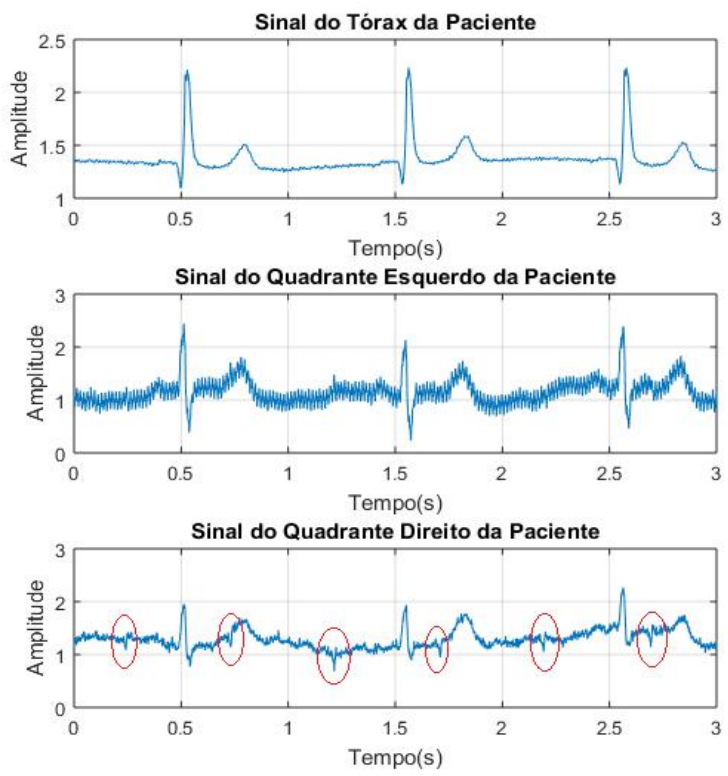

Figura 8: Espectro de frequência do sinal. Fonte: Acervo do autor.

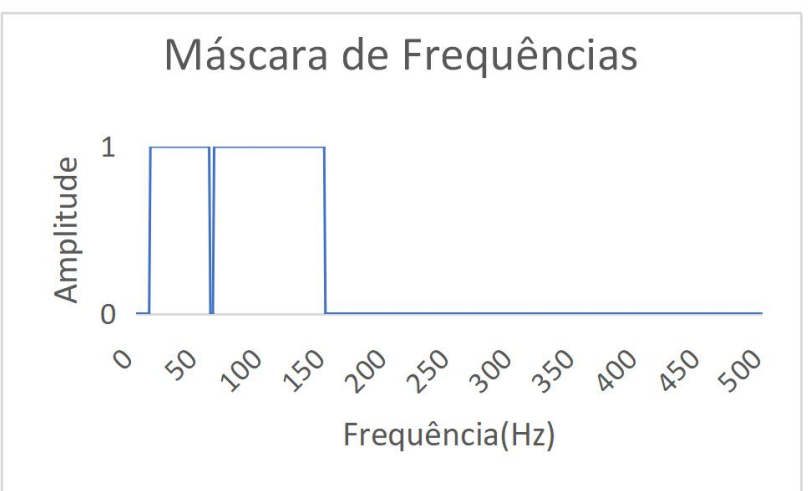

Figura 9: Máscara de frequências utilizada na etapa de filtragem.

Fonte: Acervo do autor.
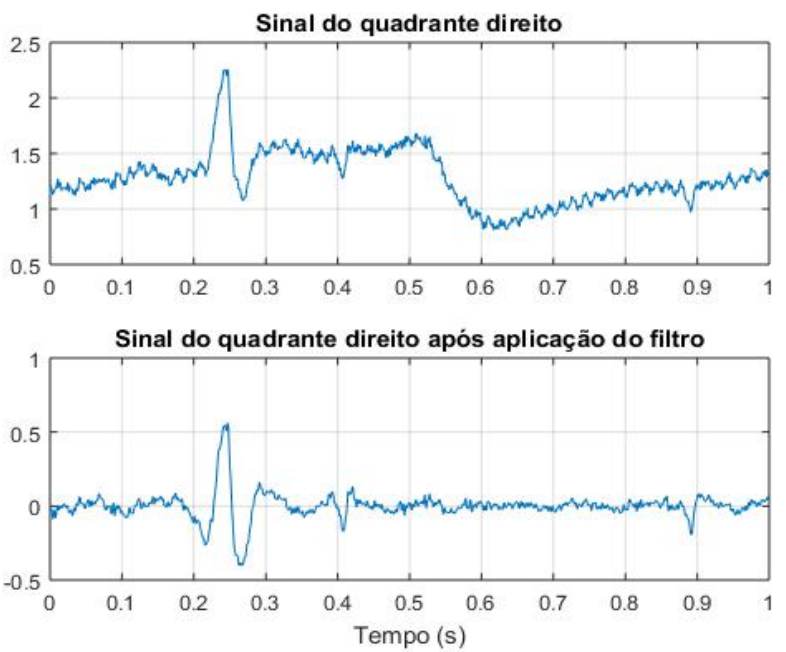

Figura 10: Sinal no domínio do tempo, antes e depois da realização da filtragem do sinal. Fonte: Acervo do autor.

A Figura 11 exibe o sinal normalizado após a filtragem e o cálculo da variância do sinal. Neste sinal pode-se identificar que o pico da variância está correlacionado com o complexo QRS do batimento materno. Os batimentos fetais apresentam uma variância pequena em relação à do complexo QRS. 

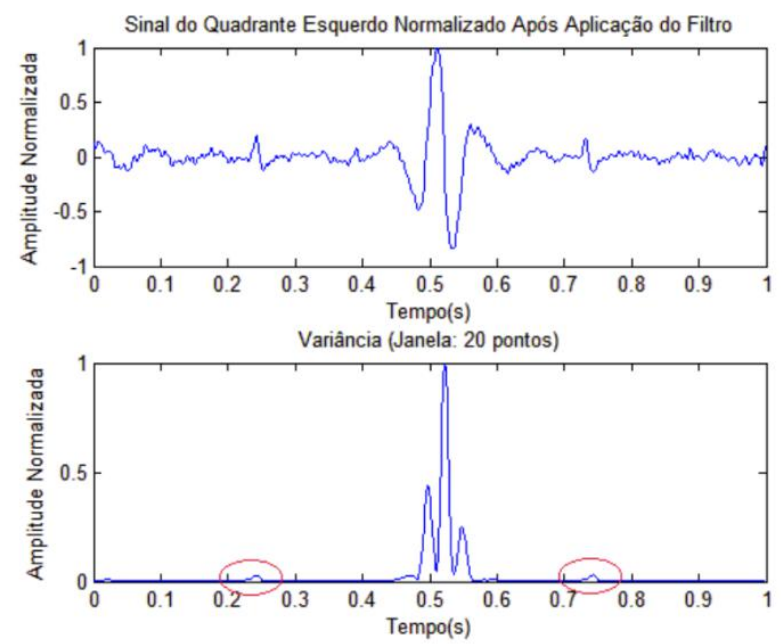

Figura 11: Sinal após a filtragem (a) e o cálculo da variância deste sinal (b).

Fonte: Acervo do autor.

Como exemplo, para a aplicação dos filtros, utilizou-se um sinal com 17 segundos de gravação. As frequências calculadas a partir do algoritmo são apresentadas na Figura 12, na qual pode-se identificar que algumas frequências são artefatos. Desta maneira, o cálculo do desvio padrão se faz necessário para identificar esses valores e retiralos do registro, deixando apenas os valores que compõem as frequências do batimento cardíaco do feto. Assim, adotou-se como ponto de corte os valores maiores do que a média somada ao desvio padrão e menores do que a média subtraída o desvio padrão, para que sejam eliminados do sinal original.

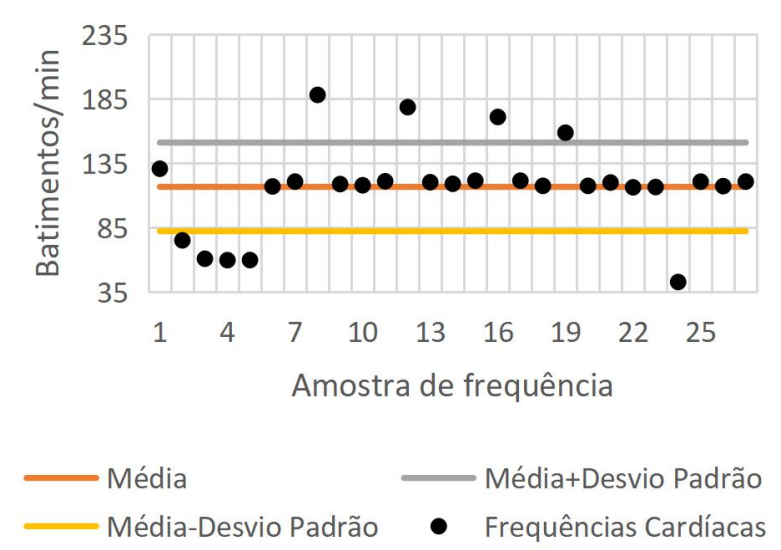

Figura 12: Frequências dos batimentos fetais calculadas.

Fonte: Acervo do autor.
Os dados calculados a partir da amostra foram iguais a:

- Desvio padrão das frequências: \pm 34 , 4187 bpm;

- Média das frequências: 119,0476 bpm;

- Limite superior $=$ Média + Desvio Padrão $=153,4663 \mathrm{bpm}$;

- Limite inferior $=$ Média - Desvio Padrão $=$ 84, 6289 bpm [19].

Os valores fora da faixa aceitável são considerados artefatos de frequência, causados por detecção de pontos falsos ou pela exclusão de alguns pontos tendo em vista que os batimentos fetais e maternos podem coincidir e que neste caso ambos serão eliminados.

Neste exemplo, após a aplicação do algoritmo que elimina os pontos fora destes limites, ficaram 18 valores de frequências dentre as calculadas inicialmente. Em seguida foram calculados os valores da nova média e do desvio padrão:

- Desvio padrão das frequências: $\pm 3,28$ bpm;

- Média das frequências: 119,52 bpm.

Estes novos valores podem confirmar que os artefatos foram eliminados, pois o desvio padrão mostra que os valores das frequências variam pouco em relação à média. Os valores após a eliminação dos artefatos estão na Figura 13.

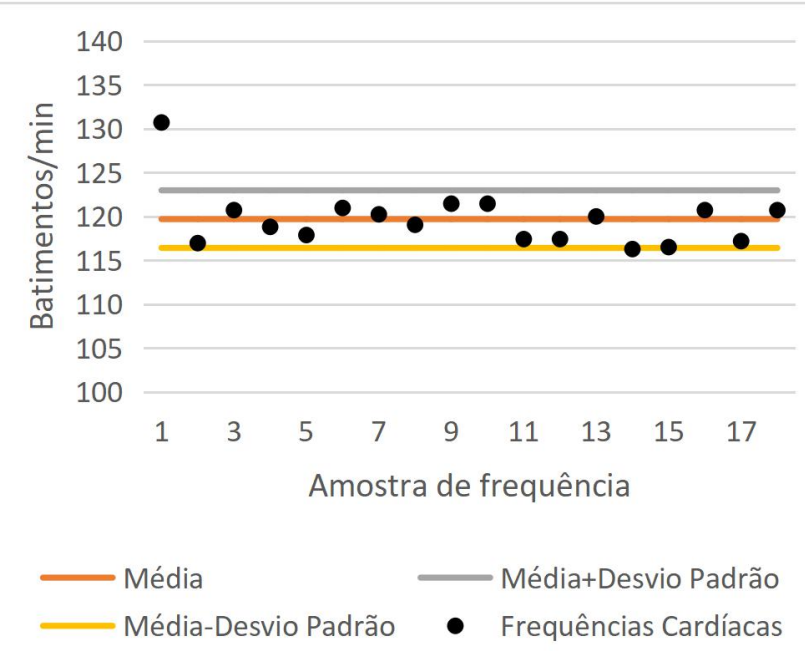

Figura 13: Frequências (bpm) após a eliminação dos artefatos.

Fonte: Acervo do autor. 
Com o objetivo de confirmar os dados, foi realizado a localização dos picos dos batimentos fetais por um médico especialista. Desta maneira, este segundo método foi considerado o padrão ouro em relação à detecção dos batimentos do feto [20, 21]. Para calcular manualmente a frequência, foi utilizada a ferramenta de gráfico data cursor do Matlab. Deste modo, com os comandos de zoom e o data cursor, foram determinados manualmente os instantes em que o pico do batimento fetal acontecia. Os valores das frequências foram calculados posteriormente e estão expostos na Figura 14. O valor médio inicial das frequências foi de 118,57 bpm com um desvio padrão de $\pm 4,51 \mathrm{bpm}$. 0 mesmo processo indicado para retirada automática dos pontos fora dos limites, superior e inferior, foi realizado com estes dados. O resultado demonstrou que 0 desvio padrão foi reduzido para $\pm 2,39 \mathrm{bpm}$ e manteve-se a média em 118,57bpm.

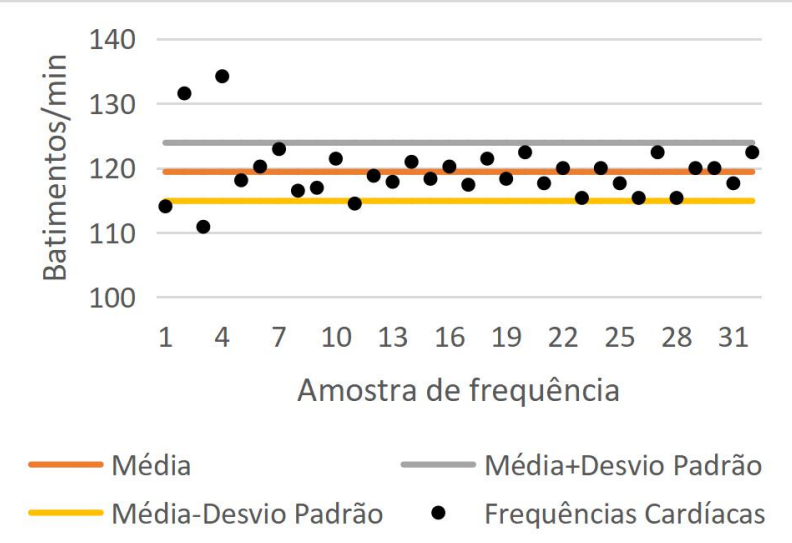

Figura 14: Valores das frequências (bpm) obtidas manualmente com o auxílio do Matlab.

Fonte: Acervo do autor.

O quadro 1 relaciona os dois métodos aplicados neste trabalho para as quatro voluntárias. Comparando os resultados encontrados, a diferença entre os valores de frequência média dos batimentos fetais em relação ao padrão ouro foi de $0,94 \mathrm{bpm}$, que representa um erro de $0,7 \%$.
Quadro 1: Comparação entre o método manual e o automático para as quatro amostras.

\begin{tabular}{|c|c|c|c|c|}
\hline & \multicolumn{2}{|c|}{$\begin{array}{c}\text { Método Manual } \\
\text { com auxilio no } \\
\text { cálculo das } \\
\text { frequências }\end{array}$} & \multicolumn{2}{c|}{$\begin{array}{c}\text { Algoritmo } \\
\text { Desenvolvido } \\
\text { neste trabalho }\end{array}$} \\
\hline Voluntária & $\begin{array}{c}\text { Valor } \\
\text { Médio } \\
\text { (bpm) }\end{array}$ & $\begin{array}{c}\text { Desvio } \\
\text { Padrão } \\
\text { (bpm) }\end{array}$ & $\begin{array}{c}\text { Valor } \\
\text { Médio } \\
\text { (bpm) }\end{array}$ & $\begin{array}{c}\text { Desvio } \\
\text { Padrão } \\
\text { (bpm) }\end{array}$ \\
\hline 1 & 118,58 & $\pm 2,39$ & 119,52 & $\pm 3,28$ \\
\hline 2 & 125,39 & $\pm 1,88$ & 126,58 & $\pm 6,31$ \\
\hline 3 & 157,08 & $\pm 2,48$ & 155,04 & $\pm 1,09$ \\
\hline 4 & 138,96 & $\pm 3,09$ & 137,64 & $\pm 4,87$ \\
\hline
\end{tabular}

Fonte: Acervo do autor.

Após todos os testes de aquisição e análise do sinal dos resultados do protótipo, passou-se para a parte de desenvolvimento de um equipamento, onde já foi desenvolvida uma placa baseada no chip MKL05Z32VFM4. A Figura 15 mostra a visualização em 3 dimensões da placa fabricada.

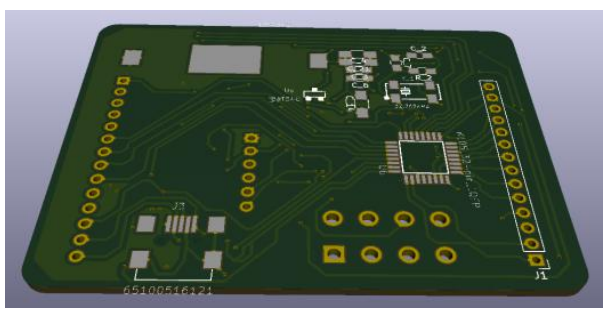

Figura 15: Visualização 3D da placa projetada. Fonte: Acervo do autor.

\section{CONCLUSÃO}

Como principal contribuição deste trabalho, tem-se o desenvolvimento de um algoritmo para detecção dos batimentos para implementação de um produto portátil e o desenvolvimento de um sistema de aquisição analógica para o sinal do batimento fetal.

Em geral, os centros obstétricos contam com poucos médicos para a monitoração de algumas dezenas de mulheres grávidas em trabalho de parto, sendo inviável a verificação dos batimentos fetais de todos os indivíduos segundo o protocolo indicado, que é a cada meia hora. Além disto, é estabelecida uma ordem de prioridade, pois as pacientes que estiverem com maior dilatação são priorizadas por estarem mais perto da hora do parto, dificultando mais ainda a verificação dos batimentos fetais de todas as pacientes, devido à falta de equipamentos.

Como resultado, foi realizada a comparação da frequência obtida com o método desenvolvido com a frequência calculada através da contagem manual de cada voluntária, utilizando as ferramentas de gráfico 
do Matlab, apresentado uma diferença entre os dois resultados menor do que $5 \%$.

Um trabalho futuro possível seria a implementação do algoritmo em um sistema embarcado já com o algoritmo de processamento implementado. Um FPGA seria ideal, pois pode executar o processamento em paralelo, possibilitando a aquisição e o processamento simultâneos.

\section{REFERÊNCIAS}

[1] DEVANE, D. et al. Cardiotocography versus intermittent auscultation of fetal heart on admission to labour ward for assessment of fetal wellbeing (Review). Cochrane

Database of Systematic Reviews. John Wiley \& Sons, Ltd, 2018. DOI: 10.1002/14651858.CD005122.pub5.

[2] SCHRAMM, K. et al. Acceptance of a new non-invasive fetal monitoring system and attitude for telemedicine approaches in obstetrics: a case-control study. Archives of Gynecology and Obstetrics,v. 298, n. 6, p. $1085-1093$, Dec. 2018. DOI: https://doi.org/10.1007/s00404-018-4918-y.

[3] SAMENI, R.; CLIFFORD, G. A Review of Fetal ECG Signal Processing; Issues and Promising Directions. Open Pacing Electrophysiol Ther J., v. 1, n. 3, p. 4-20, Jan.2010.

[4] FREEMAN, R. et al. Fetal Heart Rate Monitoring. 4. ed.Philadelphia: LWW, 2012.

[5] MAIA, M. B. Instituição, organização e profissão na assistência ao parto, em Humanização do parto: política pública, comportamento organizacional e ethos professional. Editora FIOCRUZ, 2010. DOI: https://doi.org/10.7476/9788575413289.

[6] ZHANG, S. et al. Signal Integrity Evaluation for Fetal Magnetocardiography. In:IEEEEMBS INTERNATIONAL CONFERENCE ON BIOMEDICAL AND HEALTH INFORMATICS, 75 Jan. 2012, Hong Kong. Proceeding [...] Hong Kong: IEEE, 2012. p. 253-256, v. 25.

[7] CHEN, J. et al. Fetal Heart Signal Monitoring with Confidence Factor. IEEE International Conference on Multimedia and Expo.

Proceeding [...] IEEE, jul. 2006.

[8] JEZEWSKI, J. et al. A novel technique for fetal heart rate estimation from Doppler ultrasound signal. BioMedical Engineering OnLine, v. 10, n. 1, p. 92, 2011.

[9]ANDREOTTI, F. et al. Maternal signal estimation by Kalman Filtering and Template Adaptation for fetal heart rate extraction. In: MURRAY, A. (ed.) COMPUTING IN CARDIOLOGY 2013. Zaragoza, Spain: (CinC),2013. v. 40, p. 193-196.

[10] ZHANG, N. et al. A Novel Technique for Fetal ECG Extraction Using Single-Channel Abdominal Recording. Sensors, v. 17, n. 3, p. 457,2017 . DOI: https://doi.org/10.3390/s17030457.

[11] ANDREOTTI, F. Extraction and Detection of Fetal Electrocardiograms from Abdominal Recordings.Technische Universität Dresden, 2017.

[12] PEDDANENI, H. A Comparison of Algorithms for Fetal ECG Extraction. 2004. $58 \mathrm{f}$. Thesis (Master of science) Graduate School Of TheUniversity Of Florida, Florida, 2014.

[13] TEXAS INSTRUMENTS. INA12x Precision, Low-Power Instrumentation Amplifiers. SBOS051D, Datasheet, 2018. p. 29.

[14] PERTENCE JUNIOR, A. Eletrônica Analógica: Amplificadores operacionais e filtros ativos. 8 ed. Porto Alegre: Bookman, 2014.

[15] ROBERTS, M. J. Fundamentos em Sinais e Sistemas. São Paulo, 2009.

[16] INSTRUMENTS, N. USB 6009. Disponível em:http://www.ni.com/ptbr/support/model. usb-6009.html. Acesso em: 23 outubro 2018.

[17] INSTRUMENTS, N. NI USB-6009 - Device Specifications, 2015.

[18] RODRIGUES, M. A. B. Desenvolvimento de um instrumento virtual para aquisição e análise de sinais bioelétricos. Universidade Federal de Santa Catarina, 1997.

[19] MEYER, P. L. Probabilidade: Aplicações à Estatística. 2. ed. Rio de Janeiro: Livros Técnicos \& Científicos, 1983. 
[20] ALbOLIRAS, E. T. History and Pysical Examination, em Visual Guide to Neonatal Cardiology. John Wiley \& Sons, 2018.

[21] PETERSON-BROWN, S.; JOHNSTON, T. A. Fetal Monitoring During Labour, em Dewhurst's Textbook of Obstetrics \& Gynaecology. John Wiley \& Sons, 2018. 